\title{
The Treatment of Error in Oral Work
}

\author{
John F. Fanselow
}

\begin{abstract}
Frequently, discussions about language teaching include the questions: Which types of errors should be treated and which ignored? How should errors be treated? To see the extent to which the behaviors of teachers could help answer these questions, eleven teachers were videotaped teaching the same lesson to their classes. Transcripts were made, containing both verbal and nonverbal behaviors. The analysis of the tapes showed that both the types of errors treated and the treatments used were quite similar. The teachers
\end{abstract}

seemed less concerned with errors of grammar than with incorrect meaning. Giving the right answer was the most popular treatment. The similarity of behavior among the teachers did not provide as much insight into the treatment of errors as was hoped for. The process of analysis did lead to a number of ideas about possible alternative treatments. The treatments suggested are based on the importance in learning of contrasts, redundancy, explicit feedback, and the difference between long- and short-term memory.

\section{Introduction}

Errors ESL students make, as well as speculation about the causes of errors, have been treated in a number of studies. ${ }^{1}$ Based on these studies and personal beliefs, some methodologists have made general suggestions about how teachers should treat errors. Others consider errors aberrations to be prevented at all cost and ignore their treatment. A few consider errors to be aids that show teachers how students are developing both true and false hypotheses about the language. Others do not think errors matter much one way or the other in language learning.

1. See, for example, the works by Burt and Kiparsky, Corder, Dulay and Burt, George, Holley and King, Politzer and Ramirez, Richards, and Scott and Tucker cited in the References.

John F. Fanselow (Ph.D., Teachers College, Columbia University) is Xssoclate Profesecr of Language and Education at Teachers College, Columbia University, New York, N.Y.
This study began as an attempt to see how experienced ESL teachers treated errors in their classes. The process of analyzing the data collected for the study led not only to a description of eleven teachers' treatment of error, however. It also led to ideas about preventing some types of errors and possible alternative methods of correcting other types of errors.

\section{Collection of Data}

To prepare for the collection of data, eleven experienced ESL teachers were asked to allow a technician to videotape their teaching performance. They were given the same lesson plan and materials, and asked to teach the lesson to one of their regular classes. Adjective word order and the verbs 'holding' and 'wearing' were the focus of the lesson. The teachers were told their taped lesson would be compared with other lessons; they 
were not told what aspects of the lessons were to be studied. The lessons were videotaped, and transcripts were made which included notations of non-verbal as well as verbal behaviors. The tapes and transcripts provided the data for analysis.

Counting and Judging Errors and Responses

In analyzing the data, any utterances in English made by a student, either solicited by the teacher or volunteered by the student, were considered responses; less than three percent of the responses were student initiated. When a teácher interrupted a student response to provide new or additional information, the student's continued response was counted as a separate response.

Errors were determined in two ways. When a teacher treated part of a response as incorrect, the treated part was labelled incorrect. If the teacher later in the same lesson did not treat something he had earlier judged incorrect, the part of the response that had been considered incorrect earlier was still labelled incorrect on the transcript. Some teachers asked students to change full forms to contractions in one part of the lesson and accepted full forms in another part of the lesson. Since full forms were considered errors at one point, they were labelled as errors whenever they occurred. The teachers in two classes never treated the full form, such as 'He is,' as an error. The absence of contractions in these lessons was never labelled incorrect. Likewise, some teachers consistently accepted 'wool' rather than 'woolen' before a noun; 'wool' was labelled an error only when the teacher treated it as an error.

Other errors were judged on an absolute scale. The substitution of a phoneme, such as /ow/, in the word 'glove,' even if not treated, was labelled as an error. In the sentence 'I holding glove,' the omission of the verb and the article were both noted even if the teacher did not indicate that there was anything wrong with the sentence.

One type of non-linguistic error was also noted. When a student response was correct linguistically but different from what the teacher had asked the student to do, it was labelled 'different task.' Here is an example of this type of response.

Teacher: What are you holding? (expects student to tell him)

Student: What are you holding? different task

Teacher: The answer!

Presentation and Discussion of Quantitative Data

On the average, errors occurred about every fifteen seconds. 2 The average length of the classes was twenty-one minutes; approximately sixteen responses were made each minute, and $27 \%$ of these were judged incorrect.

The errors were grouped according to area of content. Incorrect function words, such as 'he' or 'she,' or omission of the article or auxiliary verb made up $27 \%$ of the errors. Incorrect pronuncia-

\section{The type of error least}

$$
\text { likely to be treated }
$$

is a grammatical error.

tion, such as 'jello' for 'yellow' or 'beret' for 'beret,' made up another $28 \%$. Incorrect word form, which involved incorrect agreement between subject and verb or the omission of suffixes such as the 'ing' in 'hold,' made up 19\% of the errors. Incorrect word order accounted for $7 \%$ of the errors, and incorrect content words, such as 'plastic' for 'wool,' made up 12\%. The non-linguistic error of performing a different task made up $7 \%$ of the errors.

in the lessons, four major types of tasks were set: question-answer drills with 'Wh' questions, repetition of words or patterns, substitution drills,

2. Tables showing detailed tallies of all the information contained below as well as tallies of all errors and treatments by individual lesson are available from the author. 
and question-answer drills with yes/no or either/or questions. Of the 778 tasks set in which errors were made, $48 \%$ of the errors occurred in question-answer drills with 'Wh' questions, $28 \%$ occurred in repetitions, $22 \%$ in substitution drills, and $2 \%$ in question-answer drills with yes/no or ei ther/or questions.

As one might expect, errors occur a great deal less frequently when the task simply calls for one to say "yes" or "no" or repeat part of an either/or question. The fact that $22 \%$ of the errors occurred during substitution exercises and $28 \%$ occurred during repetitions suggests that substitutions are not necessarily harder than repetitions. The percentages clearly show that asking students 'Wh' questions that require the identification of objects or the recall of sentences practiced earlier seems to be one sure way to elicit errors.

To discover the ways teachers treated the errors, the behaviors which followed errors in responses were labelled in one of sixteen ways. Table I shows the sixteen types of treatment and the approximate number of instances of each.

In addition to tallying the types of treatment given after errors, the types of treatment given according to the type of error was also counted. This tally showed that the type of error least likely to be treated is a grammatical error. For example, around $33 \%$ of the errors in function words were ignored. On the other hand, if a student did a task different from the one the teacher had set or produced an incorrect content word, there was a 94\% chance that treatment of some kind would follow. Omission of an auxiliary or incorrect word order, content words, and pronunciation evoked the most instances of explicit indications that a response was incorrect. Of 133 indications of 'no,' eighty-five occurred after these errors: fifteen after omission of auxiliary, thirteen after incorrect word order, twenty-two after incorrect

TABLE 1

Types of Treatment of Errors and Approximate Number of Each Type for Eleven Classes

\section{Types of Treatment Following Error}

Approximate Number of each Type and Percentage

1. No treatment: sets another unrelated task

2. Acceptance of response containing error: "Yes, OK," shakes head 41 , (4\%)

3. Sets task again with no new information: "Again"

4. Gives correct answer orally

5. Correct response given orally by another student

6. Gives part of correct response or established cue in a different medium:

S: Wool. T: (points to an ' $n$ ' written on the blackboard)

S: He holding. T: zzz. S: Oh, he's holding.

S. A silk yellow tie. T: (switches arms) S: Yellow silk

7. Gives information: S: Woolen black beret

$$
\text { T: Color first }
$$

8. Presents alternatives: S: Where are you holding? T: Where or what?

9. Repeats response with rising intonation:

S: Leather grove $T$ : Leather what?

S: Plastics T: Plastic?

S: Green (correct answer is gray) T: Green?

10. Gives indirect information:

S: (holding a silk tie) Linen. T: The material is from China.

S: Gloves. (pointing to one glove) T: How many?

11. Stops student from continuing response:

S: I holding... T: (holds up hand like a policeman)

12. Indicates no with a gesture:

Shakes head sideways, grimaces, or shakes finger

13. Says "no" or "uh uh"

14. Gestures and says "no" or "uh in"

15. Repeats student incorrect response and says "no:" St Linen. T: No, not linen

16. Miscellaneous: student stops in mid-response and corrects self; teacher reaction unintelligible on tape; teacher simply waits, and student starts again without error

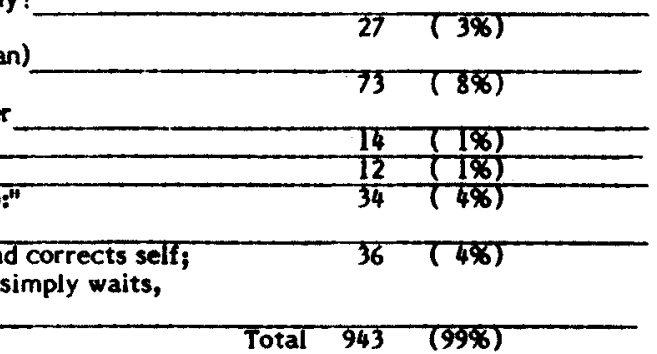


content word, and thirty-five after incorrect pronunciation.

Presentation and Discussion of Descriptive Data

Any type of content analysis or categorization obscures some information. Repeated viewing of the videotapes, coupled with intensive counting and coding of the responses on the transcripts, led to several crucial realizations. The figures do not show the fact that the treatments sometimes occurred in combinations, some of whicl, were ambiguous. After an error in a response, a teacher sometimes gestured 'no,' rubbed a sock, and pointed to a bag--three separate treatments that could be difficult to comprehend. There were times when teachers said "fine" and at the same time shook their heads sideways. The 'fine' no doubt was intended as a signal that the response was the one expected; the negative gesture was probably intended as a signal that some part of the response was incorrect.

The figures do not reflect the lack of consistency in treating errors either. In one part of a lesson, attention was focused on the absence of an

\section{Treatments sometimes}

occurred in combinations,
some of which were
ambiguous.

article or an auxiliary verb; in another part of the same lesson, these omissions were ignored.

The quantitative information also fails to show that after errors of pronunciation, short repetition cycles occurred. The teacher frequently asked each student in turn to say the word that had been mispronounced by one student; choral repetition of the mispronounced word of ten took place during these pronunciation interludes.

Since the figures are based on all the tapes, they also fail to show that some teachers did not use particular treatments at all. Though $3 \%$ of the time, errors were followed with some indication from the teacher to stop, only a few teachers used this tactic. Likewise, some teachers never said "no," presented al ternatives, or gave information.

The one tactic all the teachers did use was to present the right answer or part of the answer after an error. Rereading the transcripts of the lessons and re-viewing the tapes of the lessons with this fact in mind suggests strongly that a major goal of these lessons seemed to be to have students answer questions and use patterns in a way the teacher had planned--with no variation allowed. It is true that the teachers were asked to teach particular content. But students sometimes made side comments containing information related to the lesson; these comments were 'squelched' rather than used. Errors in adjective word order involving color and material were infrequent. Yet, when students referred to an object as cheap or dirty, or a color as dark, teachers did not try to incorporate these adjectives into the paradigm outlined in the lesson plan. No effort was made to elicit, use, or incorporate student words relating to the color-materialobject pattern.

The only time students were encouraged to try out their words was in the identification of objects:

T: What material is this? (holding a tie)

S. Linen.

S: Wool.

S: Cotton.

$T$ : It's linen.

Guessing of this kind was allowed, but students were neither allowed nor encouraged to fit new words into the patterns or to describe the same objects using a prepositional phrase rather than the color-material-object pattern, e.g., "It's a red sock made of wool." This pattern can be used as a contrasting pattern to show how a red woolen sock' and 'a red sock made of wool' are related. Hypothesis testing, experimentation with language rules, seemed absent; congruence with the teachers' expectations seemed to be the rule. Though guessing of content words took place, hypothesis testing about patterns did not. Trying 
to say what the teacher wants need not be antithetic to testing out hypotheses about how language works, but in these lessons the two seemed to work in opposition.

\section{Prevention of Errors}

Though the optimum number of errors in a lesson can probably never be determined, the learning value of some types of errors seen in these lessons is questionable. Asking a student to identify an object or the quality of an object shows whether the student knows the word or not, but yes/no and either/or questions about objects would meet his aim as well--and with fewer errors, no doubt. The teacher's presentation of content words might prevent many incorrect ones from being said, and the time saved might better be used to treat errors involving incorrect form.

In a few instances, teachers expected students to respond very quickly. Though some consider a quick pace necessary to keep a lesson moving, requiring students to respond more quickly than one would be expected to in ordinary conversation has drawbacks. Native speakers make slips of the tongue during verbal games and ordinary conversations in which there is a great deal of quick give and take. With a longer 'wait time,' some students may be able to edit their responses in their mind before producing them. As a result, some of those errors that are the result of slips of the tongue might be prevented. In one descriptive study, English teachers were found to vary the amount of lead time they gave according to the academic level of the class; much less lead time was given to students in below-average classes than in aboveaverage classes. ${ }^{3}$ The salutary effect of a longer wait time on both the quality and length of student responses was described in a study of science classes. ${ }^{4}$

3. James Hoetker, "Teacher Questioning Behavior in Nine Junior High School English Classes," Research in the Teaching of English, 2, ii (1968), 99-106.

4. Mary Budd Rowe, "Science, Silence, and Sanctions," Science and Children, 6, vi (1969), 11-13.
A good deal of time in these lessons was spent distinguishing the meaning of 'wearing' and 'holding' through questions such as "What's she doing?" When I have a sock over my hand, am I wearing it or holding it? If I put a small boxing glove on my finger, should I use 'holding' or 'wearing?' These subtle distinctions are difficult for native speakers. Before asking someone to tell what someone else is doing in a particular instance, the teacher might indicate which word he thinks goes with the particular action. After giving an indication of the word to use, the student

\section{Some teachers did not use particular treatments at all.}

might be tested. To expect students to make ambiguous word choices is simply to encourage errors that do not have much learning value.

To decrease the number of responses that are different from those expected or contain fewer words than desired, explicit directions with examples and a longer wait time before the response might be helpful. The teachers in many of these classes tended to indicate implicitly the kind of responses desired, and after the responses rather than before. When a student says "You are holding a white hat" rather than "You are holding a white woolen hat" in answer to the question "What am I holding?," he does not necessarily lack language ability. The answer is perfectly natural with or without a word for the material. To wait until after the response to say that you want the material as well as the color will probably cause more undesired responses than saying it before the response.

Though errors in grammar and pronunciation cannot be prevented, repetition of them might be. Many cycles in these classes contained instances of repeated errors due in part, perhaps, to the fact that the teacher of ten did not indicate either that an error had occurred or where it had occurred. 
When a student's incorrect response is followed by a subtle shaking of the head or "again," the student does not necessarily know that his response contained an error or know the location of the error. 'Again' can mean "I did not hear you" as well as "you made a mistake." Shaking one's head sideways--the method used to communicate $55 \%$ of the 'no's' in these lessons--while students are engaged in looking at fellow students to whom they had just asked questions or looking at the materials in their hands on which they had just commented, is probably not easy for the student to see. These types of rather ambiguous reactions probably caused some errors to be repeated to no purpose. The value of this type of error repetition is questionable:

S: He holding hat.

T: Again.

S: She holding hat.

$T$ : She?

S: holding...

Even the presentation of the right answer after an incorrect response can lead to repetition of the error. In these lessons, the teachers presented the right answer after correct as well as incorrect responses. This example illustrates one type of pattern of treatment.

$T:$ It's blue.

$S_{1}:$ It blue.

$\mathrm{T}$ : It's blue.

$S_{2}$ : It's blue.

$T$ : It's blue.

$S_{1}$ : It blue.

$T$ : It's blue.

$S_{1}:$ It blue.

The teacher's purpose for the repetition in lines 3, 5, and 7 may be different. Line 5 may be intended as reinforcement. Lines 3 and 7 may be intended as signals that errors have been made. But since the same behavior has at least two separate purposes, the students' perceptions of the behavior may be different from the teacher's intentions. These types of behaviors can be considered ambiguous.

Another type of ambiguity might have occurred as a result of the inconsistency of the teachers in treating errors. Teachers accepted incorrect responses in one part of a lesson and did not accept them in other parts of the lesson. Consistency in standards of acceptance, explicit information about types and locations of errors, explanations to show what is expected before responses rather than after, longer wait times, and testing of knowledge of content words by yes/no and either/or questions rather than question-word questions might all help prevent some types of errors. Explicit information and consistency of standards may also aid students in developing criteria of correctness.

Suggested Alternate Treatments

Basically, the treatments in these lessons involved giving the students another chance, with or without various amounts of information; either the complete answer was given or a part of the answer was given in speech or in some other medium. Errors were treated by representing the complete correct response or the part of the response needed by the student to make his response correct. The degree to which this basic type of treatment helps move patterns into students' long-term memories or helps them establish deep-level rules or categories is probably minimal.

One al ternative approach would be to present a number of tasks after errors are made in order to help students establish categories, al ter their deep rules for generating utterances, and help move patterns into long-term memory. When the answer alone is given, a sense that the language works in a patterned way cannot be easily discerned. In the follow-up tasks suggested here, the goal is to help students move the patterns into long-term memory, establish categories, alter deep-level rules, and point out relationships between different patterns in the language. These tasks may not produce the right answer as quickly as the teacher's giving the answer would. However, their goal is to teach rather than just correct. They are not suggested for treating incorrect content words or slips of the tongue. They are suggested for 
treating errors resulting from incorrect hypotheses about how the language works and involving problems of perception as well as production.

The purpose of these tasks is to reduce students' uncertainty about how the language works. The assumptions underlying them are: (1) We learn what something is by what it is not; (2) Redundancy can aid learning; (3) Explicit feedback that indicates whether something is wrong as well as how it is wrong can help develop understanding; (4) What ears hear and eyes see is not necessarily what is said or written; our prior knowledge colors our

\section{Experimentation with language}

rules seemed absent;

congruence with the teachers'

expectations seemed to be the

rule.

perceptions and blinds us to certain critical distinctive features. ${ }^{5}$ The tasks are not new; a few were used incidentally in these lessons, and most have been suggested in methods books, though not necessarily for use in the treatment of error.

One type involves discrimination exercises, reserved by many for minimal-pair drills in pronunciation lessons.

(1) S: I holding a white woolen hat.

$T$ : I holding, I'm holding. Are these the same?

(2) T: (pointing to I'm and I am on the blackboard) Which one of these is the full form?

(3) $T$ : I holding, I'm holding. Which is incorrect?

(4) T: I holding, correct or incorrect?

Another type of task involves classification. For example, the teacher draws three columns on

5. Frank Smith, Understanding Reading (New York: Holt, Rinehart and Winston, 1971). the blackboard and puts labels on them:

\begin{tabular}{|l|l|l|}
\hline Full Form & Contraction & Incorrect \\
\hline & & \\
& &
\end{tabular}

The students copy the columns with the labels. The teacher then dictates models, and students write each in the proper column. The teacher gives immediate knowledge of results by stating the column each belongs in immediately after he states the model. There is no need to limit the models to 'I' or even to real words. Models such as 'John's holding,' 'Jim holding,' 'I lopping' can also be used. The form is what is being highlighted.

The goal of all of these types of tasks is to help students set up categories in their minds. Whether students are asked to discriminate between two different correct forms or between incorrect and correct forms, or whether students are asked to classify many examples of different forms, the goal is the same-to help students develop categories by seeing not only what models contain but also what they do not contain, so ambiguity about the language can be reduced.

Tasks involving analysis can also be set. The teacher can write examples on the blackboard and ask questions about each example that highlight distinctions.

T: (writes I'm holding and I am holding on the board) How many syllables in the first one?

S: Two.

T: Spell the second one.

S: (spells the words)

$T$ : What sound is between 'I' and ' $h$ ' in the first? In the second?

The goal of these tasks is to provide explicit information about the specific aspect of the language causing difficulty.

Finally, manipulation exercises can be conducted to show fur ther contrasts and relationships.

T: Who's holding the ski cap? (said with eyes closed)

S: I am.

T: What're you doing with the ski cap now?

S: I'm passing it to Clara. Now she's holding it.

T: Clara, you're holding it now.

S: No, I'm wearing it now. 
T: I don't believe you. I think it's too small for you.

S: Well, I am.

Even mechanical manipulation exercises might be used to help show relationships, using either real or nonsense words.

T: I am waiting. Make a contraction.

S: I'm waiting.

$\mathrm{T}$ : Bin is binning. Make a contraction.

S: Bin's binning.

Teachers may wish to make use of redundancy, contrasts, and explicit information in their feedback as well as in the tasks they set for their students to perform. Teachers can categorize, analyze, and manipulate content in their reactions to errors as well as in their stimuli.

These examples show a teacher categorizing.

S: I holding the bag.

T: Not 'I holding the bag.' I'm holding the bag. 'I holding' is wrong. I want you to say 'I'm holding.'

S: I holding the bag.

T: I holding, I'm holding--these are different.

Here are exarnples of a teacher analyzing in his reactions.

S: I holding a bag.

T: We put a sound between 'I' and 'holding' like this: I'm holding. With the sound, I'm holding. Without the sound, I holding.

S: I holding a bag.

T: You said $\mathrm{i}-\mathrm{h}-\mathrm{o}-\mathrm{l}-\mathrm{d}-\mathrm{i}-\mathrm{n}-\mathrm{g}$; in English, we say $i-m-h-o-1-d-i-n-g$.

A reaction might even contain some manipulation of language.

S: I holding a bag.

T: I am holding a bag. I'm holding a bag. Am I holding a red bag? Yes, I am. I'm holding a red bag. Is he? Yes, he is too. He's also holding one.

The tasks and feedback involving analysis, categorization, and manipulation need not be done orally. Students can be asked to write the information requested in regular or thography or in phonetic script; teachers can also present the language for analysis, categorization, or manipulation in these media. Using media other than speech not only contributes to redundancy but is also more explicit than speech for some people; seeing language in black and white helps some hear it.

Some argue vehemently against making use of errors that occur during teaching. If a student has been using an incorrect form for months, a few additional productions of the incorrect form would seem unlikely to set the pattern any more firmly in his mind. Many students have never seen or heard the distinctions between incorrect and correct forms, and some probably cannot without some assistance. Incorrect forms themselves can be avoided by humming the examples or using nonsense words to represent them. The correct sentence 'He's wearing a hat' could be rendered by 'emz emming e em.' The incorrect sentence 'He

\section{The goal is to help students develop categories by seeing not only what models contain but also what they do not contain.}

wearing a hat' could be rendered by 'em emming e em.' Vocalization or the use of nonsense words might help students discriminate and classify without exposing them to the error itself.

These tasks can be done on the spur of the moment or some time after an error is made. If the teacher or student 'secretaries' write the errors down for future treatment, two advantages result. First, the form is presented in another medium, important to those who believe in the value of redundancy; second, the teacher is given time to reflect on the cause of the error. Sequencing tasks involving analysis, categorization, and manipulation, based on errors, requires some insight into the students' mistaken hypotheses. The length of time needed to prepare tasks will no doubt decrease with experience. As teachers become more facile with setting the types of tasks suggested here, the 'time out' between recording the error and its treatment may decrease from overnight to a few seconds. 
As students see the new pattern of treatment, they may begin to use it. Students in the lessons in this study treated errors that their fellow students made just as the teachers did. As teachers alter their patterns of treatment, students might also.

Though a great number of different tasks and separate combinations of feedback can be generated that involve analysis, categorization, and manipulation, they can all be done in a few seconds, and time spent doing them is probably at least as well spent as time spent giving answers alone. These tasks and these types of feedback give the students a chance to see relationships; given contrasting examples, they can begin to form rules.

Students' categories and relationships may be different from ours; we do not know what is going on in the students' minds. But the tasks and feedback suggested here do give the minds a chance to work. Categorizing and analyzing might not only help students correct one incorrect utterance; they might also help students establish rules at a deeper level. No attempt is made to ask the student to talk about the categories he develops; indeed, the point is that he simply be given a great deal of information in 'bits' so that he can develop categories and see relationships. Few teachers can describe the rule for the pronunciation of the past tense forms in English before their first phonetics course; they have, however, established categories at a deeper level that allow them to produce the correct forms. On Sesame Street, viewers are shown multiple examples of language in many media; the examples are commented on and contrasted; the amount of time spent on each point is extremely small. The tasks and feedback suggested here are related to many of the kinds of tasks and feedback presented on Sesame Street.

Even if these types of tasks or feedback do not develop deep-level rules or help move patterns into long-term memory, they do force careful listening on the part of the students as well as the teacher. Teachers ignored $18 \%$ of the errors and accepted $4 \%$ in the lessons studied here. They treated some errors one minute and ignored them the next. Whether they chose to, or did so because

\section{Errors are part of \\ learning--mistaken hypotheses \\ and wrong connections are \\ normal.}

they heard what they wanted and expected, and filled in what they did not hear in their mind, we do not know. Yet, these teachers expected students to hear well enough to distinguish their own incorrect response from the teacher's subsequent corrected repetition of their responses. If students are to hear these differences and learn from them, training in listening is vital. If teachers are to hear more clearly what their students are saying, training in listening is vital for them, too. Tasks and feedback requiring analysis, categorization, and manipulation force careful listening discrimination and may also help teachers and students develop consistent criteria of acceptance.

An even more critical reason for using these types of tasks and feedback might exist: they both suggest that errors are part of learning--mistaken hypotheses and wrong connections are normal. While giving an answer to a student may communicate the message "You are wrong; you should not leave out words," these tasks may communicate the message "Errors provide an important springboard for lessons; they are normal. Your hypotheses about how the language works are the only means $I$, the teacher, have to find out what you need to know."

There are those who suggest another approach to treating errors: ignore all errors except those that prevent the listener from receiving the message being practiced or communicated in the students' utterances. The teacher ignores all errors unless he does not understand what the student is trying to say. In this approach, the teacher treats the breakdown in communication the same way we treat such breakdowns in conversations. We say "I beg your pardon," or we repeat 
the utterance with rising intonation in such a way as to communicate the message "Is that what you said?"

Advocates of this method make these points:

(1) Children and adults who learn languages by immersing themselves in another culture seem to learn this way; (2) Shopkeepers, playmates, fellow passengers on buses, and bar companions are not trained to set tasks or provide explicit feedback when people learning their language mispronounce words or leave out function words. On the other hand, they do not ask those learning the language to identify objects either; they identify objects for the learners when the learners ask the names for the objects. Though systernatic analysis would need to confirm this observation, many learners as a matter of course ask bartenders, shopkeepers, and others for explicit information. Those 'learning on their own' can be heard asking "Is this correct?" "Is this a or a ?"

"Please write that word."

"How many syllables does that have?" These questions show learners trying to learn from contrasts and redundancy as well.

\section{Conclusion}

The purpose of this study was primarily to try to describe the treatments eleven experienced second language teachers used in treating oral work. The fact that certain patterns seemed to be prevalent, while others rare, led to a discussion of types of tasks and feedback teachers might use in their teaching in addition to those that seemed common--at least to this small group of teachers.

Whether the teachers in this study did what should be done or not, we do not know. After viewing the tapes and discussing the issues presented here, the teachers saw how what they had done in their classes differed from what they thought they had done. Seeing what they had done and abstracting what they had done through coding gave them a clearer perception of their treatment of error as well as the way they and the students used language. Options appeared to them that might otherwise not have without systematic coding and analysis.

A great many questions remain unresolved. Teachers might be interviewed to see what they consider their hierarchy of errors to be; their treatment of errors they consider serious could be compared with their treatment of errors they consider less serious. The rationale for the kinds of priorities teachers have for correcting errors might be studied as well. We have to examine teachers teaching their own lessons rather than a lesson presented to them; we have to look at the same teachers at different times to see if different lessons or different class sizes bring out different treatments. We could look at lessons through 
different 'Ienses' than the one used in this study and develop different categories of errors and treatments. We could also ask teachers to treat only those errors which had caused a breakdown in communication, to treat errors in some lessons only by saying "wrong, try again" and giving the answers and in others by giving a longer wait time, explicit feedback, and tasks that force listening. We can also simply ask teachers and students the types of treatments they prefer and why they prefer them.

This study has been simply a pilot, an initial probe into the definition of the problem. The effects the treatments outlined and described here have on students' errors, students' attitudes toward errors, and students' feelings about taking risks in learning need to be examined. A great deal remains to be learned from studying the patterns of practicing teachers treating errors. In addition, observation of language learning outside of our classrooms, in everyday give-and-take between people trying to learn another language and those who speak the target language, may provide helpful insights. We spend a great deal of time studying linguistic forms and patterns. At least as much time can be profitably spent in examining the behavior patterns of language learners and teachers in classrooms and in the world.

\section{Summer 1978 Workshop Announced}

An institute/workshop for teachers of French will be offered by the University of Washington during the summer of 1978 . 'Teams' of French, social studies, or art/music teachers and curriculum specialists from the same school will be invited to participate. Stipends from the National Endowment for the Humanities will cover room and board and travel expenses. The purpose of the institute/workshop is to improve the participants' knowledge of contemporary French culture and assist them in developing materials and plans needed for teaching cross-disciplinary classes. For further information write to:

Professor Victor E. Hanzeli

University of Washington

GN-60

Seattle, WA 98195 .

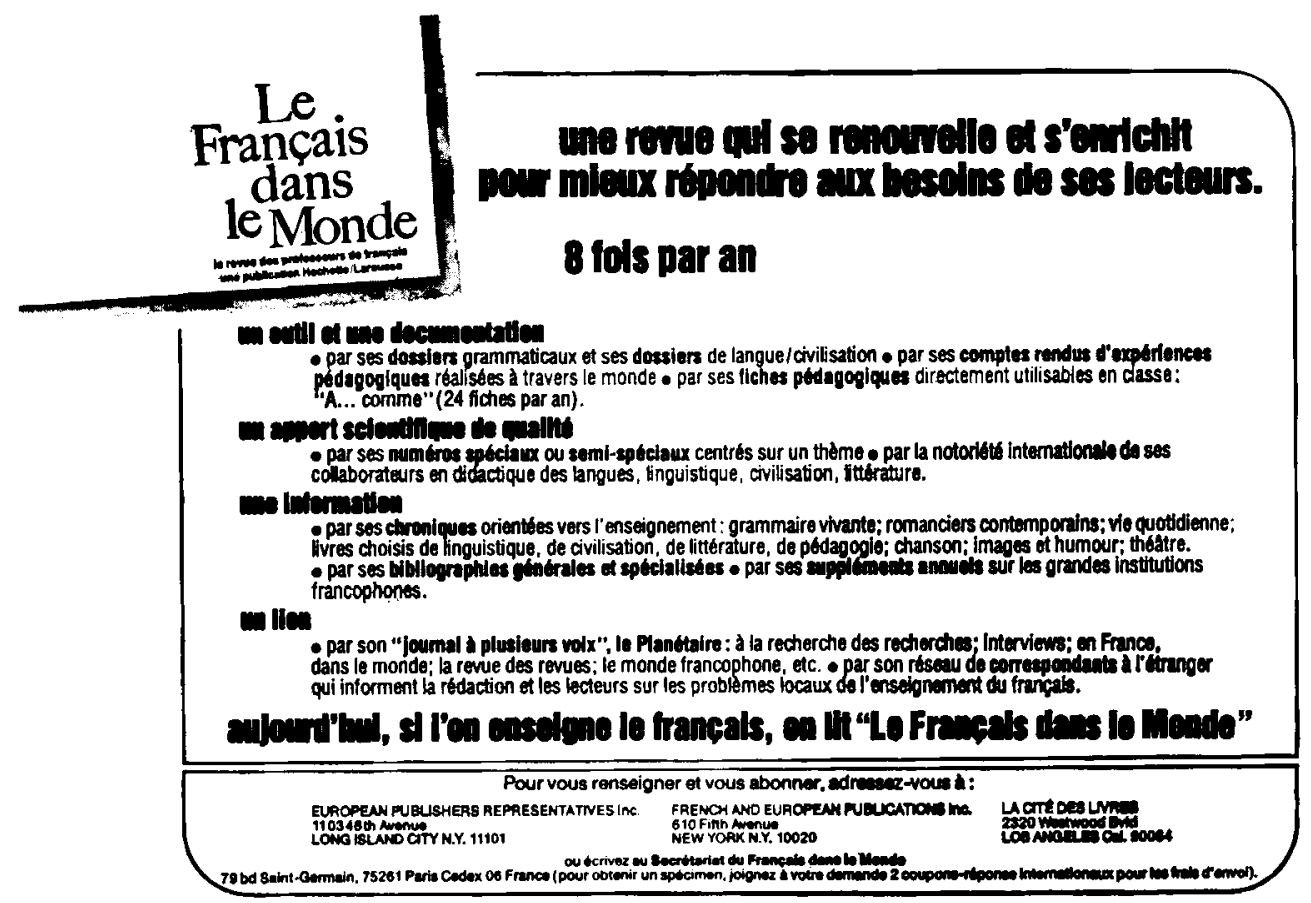

